\title{
An Adaptive Approach to Sensor Bias Fault Diagnosis and Accommodation for a Class of Input-Output Nonlinear Systems
}

\author{
Christodoulos Keliris, Marios M. Polycarpou and Thomas Parisini
}

\begin{abstract}
This paper presents an adaptive sensor fault diagnosis and accommodation scheme for multiple sensor bias faults for a class of input-output nonlinear systems subject to modeling uncertainty and measurement noise. The proposed scheme consists of a nonlinear estimation model that includes an adaptive component which is initiated upon the detection of a fault, in order to approximate the magnitude of the bias faults. A detectability condition characterizing the class of detectable sensor bias faults is derived and the robustness and stability properties of the adaptive scheme are presented. The estimation of the magnitude of the sensor bias faults allows the identification of the faulty sensors and it is also used for fault accommodation purposes. The effectiveness of the proposed scheme is demonstrated through a simulation example.
\end{abstract}

\section{INTRODUCTION}

In recent years various fault diagnosis schemes have been proposed for detecting, isolating and accommodating faults for various classes of linear and nonlinear systems (see, for instance, [1]-[5] and references therein). One of the techniques that has been used for fault diagnosis is adaptation and learning. Since the first work on proposing a learning approach for fault diagnosis [6], such techniques have been widely used for learning the modeling uncertainty to enhance fault detectability (i.e. [7], [8]) and for learning the fault function to achieve fault isolation and identification (i.e. [4], [9], [10]). Moreover, learning algorithms have also been used to facilitate the identification of the fault type, that is whether the fault is a process or a sensor one, by learning the potential fault function that has occurred and, providing an estimation of the fault function so that it can be used in fault accommodation schemes (i.e. see [7] and references therein). Specifically, various fault accommodation schemes have been considered in [11], [12].

Generally, the identification of multiple sensor faults in nonlinear systems is a very challenging task. Among the various methods employed for sensor fault diagnosis are model based-ones using either a nominal mathematical model of the system (quantitative) [7], [13]-[16] or qualitative system representation [17].

C. Keliris and M. M. Polycarpou are with the KIOS Research and Innovation Center of Excellence and the Department of Electrical and Computer Engineering, University of Cyprus, Nicosia 1678, Cyprus (Email: keliris.chris@gmail.com, mpolycar@ucy.ac.cy). Thomas Parisini is with the Dept. of Electrical and Electronic Engineering, Imperial College London, UK, with the KIOS Research and Innovation Center of Excellence, and also with the Dept. of Engineering and Architecture, University of Trieste, Italy (Email: t.parisini@gmail.com). This work was partially supported by the European Union Horizon 2020 research and innovation program under grant agreement No. 739551 (KIOS CoE).
This work falls in the class of quantitative methods for sensor bias fault diagnosis. Regarding the system representation, in previous works [10], [14], the nominal system nonlinearity function was considered as a function of output variables. More recently, in [18], [19], the system nonlinearity is broken into two terms, one comprising of output variables and the other based on the overall state vector (which may contain unmeasurable variables). Based on the assumption that there exists a suitable state transformation, fault diagnosis can be conducted for the system under the new coordinates. The work in this paper extends these results by considering a more general system formulation, in which the nominal system nonlinearity is a function of the state vector (not of the output variables).

Moreover, in earlier works [14], [18], the case of single sensor faults was considered and individual estimation models utilizing learning were designed for each potential sensor fault, so that the identification of the faulty sensor could be made on an exclusion-based logic. As it is the always the case with this approach, there is the risk of non-conclusive fault isolation due to the inability to exclude potential faults. Recently, in [8] the problem of multiple sensor fault detection and isolation was investigated for the same class of systems considered in this paper and the use of learning was made for enhancing fault detectability by estimating the modeling uncertainty. However, the task of sensor fault isolation is based on a different approach than in this work and relies on decomposing the sensing system (matrix $C$ of the output variables) into smaller sensing subsystems (with matrices $\left.C^{(I)}\right)$ and constructing all the possible combinations of these sensing subsystems (along with their respective estimation models) such that the pair $\left(A, C^{(I)}\right)$ is observable. As a result, by the use of a combinatorial decision logic the faulty sensors can be identified either uniquely or at least in smaller sensor sets. More details regarding the design and analysis of the methodology for detecting and isolating multiple sensor faults under that framework can be found in [16]. In addition, in [20] a distributed adaptive estimation scheme for isolation of sensor faults is presented, for a specific class of systems (multizone HVAC systems) with full-state measurements. Moreover, in our previous recent work [7], a fault diagnosis approach for process and sensor faults was designed by utilizing the filtering framework devised in [5] and by considering again full-state measurements.

This work, is differentiated from the aforementioned works, in the following ways: a) the problem of multiple sensor bias fault estimation for a more general class of inputoutput nonlinear systems is addressed, b) the sensor faults 
identification is based solely on the sensor bias fault estimation obtained from the adaptive fault diagnosis scheme and c) a fault accommodation scheme is presented allowing for fault tolerant operation. More specifically, regarding points b) and c), once a fault is detected, the adaptive approach is initiated with the task of approximating the magnitude of all potential sensor faults (by considering all sensors as potentially faulty). The robustness and stability properties of the adaptive scheme are presented. Based on the estimation of the magnitude of the sensor bias faults, the identification of the faulty sensors can be made and a fault accommodation scheme is presented.

The paper is organized as follows: the problem formulation is given in Section II, in Section III the details of the nonlinear estimation model are presented and, in Section IV a detectability condition that characterizes the class of detectable faults is derived. In Section V, the stability and performance properties of the proposed adaptive scheme are presented. The fault accommodation strategy is presented in Section VI and, in Section VII a simulation example is demonstrated. Finally, in Section VIII some concluding remarks are provided.

\section{PROBlem Formulation}

Consider a nonlinear dynamic system described by:

$$
\Sigma:\left\{\begin{array}{l}
\dot{x}(t)=A x(t)+f(x(t), u(t))+\eta(x(t), u(t), t)(1) \\
y(t)=C x(t)+\xi(t)+B^{y}\left(t, T^{y}\right) \sigma(t),
\end{array}\right.
$$

where $x \in \mathbb{R}^{n}, u \in \mathbb{R}^{m}$ and $y \in \mathbb{R}^{p}$ are the state, input and measured output vectors respectively, the matrix $A \in \mathbb{R}^{n \times n}$ and the function $f: \mathbb{R}^{n} \times \mathbb{R}^{m} \mapsto \mathbb{R}^{n}$ represent the known (nominal) function dynamics and $\eta: \mathbb{R}^{n} \times \mathbb{R}^{m} \times \mathbb{R}^{+} \mapsto \mathbb{R}^{n}$ represents the unknown modeling uncertainty. The matrix $C \in \mathbb{R}^{p \times n}$ is the known nominal output matrix and the vector $\xi \in \mathcal{D}_{\xi} \subset \mathbb{R}^{p}\left(\mathcal{D}_{\xi}\right.$ is a compact set) represents the measurement noise. The state vector $x$ is considered unknown whereas the measurements $y$ are known. The term $B^{y}\left(t, T^{y}\right) \sigma(t)$ characterizes the time-varying magnitude of the sensor faults. More specifically, each component $\sigma^{(k)}(t)$, $k=1,2, \ldots, p$, of the sensor fault vector $\sigma: \mathbb{R}^{+} \mapsto \mathbb{R}^{p}$, represents the time varying bias due to a sensor fault that occurs in the $k$-th sensor and the diagonal matrix $B^{y}\left(t, T^{y}\right) \in \mathbb{R}^{p \times p}$ represents the time profile of the faults, i.e.: $B^{y}\left(t, T^{y}\right)=$ $\operatorname{diag}\left(\beta^{y}\left(t-T_{1}^{y}\right), \ldots, \beta^{y}\left(t-T_{p}^{y}\right)\right)$, where $T_{k}^{y}$ is the time of occurrence of the $k$-th sensor fault and $T^{y} \triangleq\left[T_{1}^{y}, \ldots, T_{p}^{y}\right]^{\top}$. Each time profile component $\beta^{y}$ represents the time profile of each sensor fault. In this work, we consider only the case of abrupt sensor faults, and therefore the function $\beta^{y}$ takes the form of a step function:

$$
\beta^{y}\left(t-T_{k}^{y}\right)=\left\{\begin{array}{ll}
0 & \text { if } t<T_{k}^{y} \\
1 & \text { if } t \geq T_{k}^{y}
\end{array} \quad k=1, \ldots, p .\right.
$$

Without loss of generality and, in order to simplify the notation and presentation, we consider that all the sensor faults occur at the same time $T_{0}^{y}$. In the general case, in which the sensor faults do not occur simultaneously, the results contained in this paper still hold, by considering $T_{0}^{y}$ as the time of the first sensor fault, i.e. $T_{0}^{y} \triangleq \min \left(T_{1}^{y}, \ldots, T_{p}^{y}\right)$ and replacing $\sigma(t)$ with $B^{y}\left(t, T^{y}\right) \sigma(t)$ in what follows.

In this paper, we do not deal explicitly with the control design problem, and therefore, the formulation is independent of the controller used, which may either be a generic one achieving some desired control objectives. The notation $|\cdot|$ used in this paper denotes the Euclidean 2-norm for vectors and, the matrix norm induced by the 2-norm for matrices. In addition. the following assumptions are used:

Assumption 1: The state variables $x$ and the local input $u$ remain bounded in some region of interest $\mathcal{D}=\mathcal{D}_{x} \times \mathcal{D}_{u} \subset$ $\mathbb{R}^{n} \times \mathbb{R}^{m}$ (which is a compact set), before and after the occurrence of a fault (well-posedness).

Assumption 2: The pair $(A, C)$ is detectable.

Assumption 3: The modeling uncertainty $\eta$ is an unstructured and possibly unknown nonlinear function of $x, u$ and $t$ but whose norm is bounded by a known positive functional $\bar{\eta}(y(t),(u(t))$ :

$$
|\eta(x(t), u(t), t)| \leq \bar{\eta}(y(t),(u(t)),
$$

for all $t \geq 0$ and for all $(x, u) \in \mathcal{D}$, where the region $\mathcal{D}$ is a known compact set.

Assumption 4: The function $f(x, u)$ satisfies the local Lipschitz condition:

$$
\left|f\left(x_{1}, u\right)-f\left(x_{2}, u\right)\right| \leq \lambda_{f}\left|x_{1}-x_{2}\right|
$$

where $\lambda_{f}$ is the Lipschitz constant for the function $f(x, u)$ with respect to $x$ in the region $\mathcal{D}_{x}$.

Assumption 5: The rate of change of the sensor bias $\sigma^{(k)}(t)$ is unifromly bounded as follows:

$$
\left|\dot{\sigma}^{(k)}(t)\right| \leq \psi \quad \forall \quad k=1, \ldots, p,
$$

where $\psi$ is an unknown positive scalar.

Assumption 2 is required for the design of the estimation model. Assumption 3 characterizes the class of modeling uncertainties being considered as it is required to discriminate between the effects of the modeling uncertainty and the fault. Assumption 5 assumes a bounded rate of change of each sensor bias fault which is not required to be known. This is required for the stability analysis of the adaptive scheme. Of course, it is a reasonable assumption since some a priori information regarding the bias evolution can be used to determine such a bound if needed.

\section{AdAPTIVE FAULT DIAGNOSIS ARCHITECTURE}

In this Section, we explain in detail the architecture of the proposed fault diagnosis scheme which is robust with respect to the modeling uncertainty and the measurement noise. The proposed scheme consists of a nonlinear estimation model, in which under healthy mode of operation, the output estimation error is always bounded by the detection threshold and thus, no false-positive alarms are guaranteed. Once a fault is detected, an adaptive scheme is initiated to determine the potential sensor bias faults for all sensors by estimating their 
magnitudes. The estimation model is given by:

$$
\begin{aligned}
& \dot{\hat{x}}_{s}(t)=A \hat{x}_{s}(t)+f\left(\hat{x}_{s}(t), u(t)\right)+L \epsilon_{s}^{y}(t)+\Omega(t) \dot{\hat{\sigma}}(t) \\
& \dot{\Omega}(t)=A_{0} \Omega(t)-L \\
& \hat{y}_{s}(t)=C \hat{x}_{s}(t)+\hat{\sigma}(t) \\
& \dot{\hat{\sigma}}(t)=\mathcal{P}_{\sigma}\left(\Gamma^{y}(C \Omega(t)+I)^{\top} D\left[\epsilon_{s}^{y}(t)\right]\right)
\end{aligned}
$$

where $\epsilon_{s}^{y}(t) \triangleq y(t)-\hat{y}_{s}(t)$ denotes the output estimation error, the matrix $L \in \mathbb{R}^{n \times p}$ is selected such that $A_{0} \triangleq A-$ $L C$ is Hurwitz (according to Assumption 2 this is always feasible), $\Gamma^{y} \in \mathbb{R}^{p \times p}$ is a symmetric and positive definite learning rate matrix, $I \in \mathbb{R}^{p \times p}$ is the identity matrix and $\hat{\sigma} \in \mathbb{R}^{p}$ denotes the sensor fault estimation. Finally, the initial conditions are $\hat{x}_{s}(0)=0, \Omega(0)=0$ and $\hat{\sigma}(0)=0$. In the sequel we denote the state estimation error as $\epsilon_{s}^{x}(t) \triangleq x(t)-$ $\hat{x}_{s}(t)$. The projection operator $\mathcal{P}_{\sigma}$ restricts the estimation vector $\hat{\sigma}(t)$ in a predefined and convex region $\Theta_{\sigma} \in \mathbb{R}^{p}$ in order to guarantee stability of the learning algorithm in the presence of noise and modeling uncertainty. Here, $\Theta_{\sigma}$ is considered to be a hypersphere of radius $M_{\sigma}$, and hence the adaptive law for $\dot{\hat{\sigma}}$ can be expressed as [14], [21]

$$
\begin{aligned}
\dot{\hat{\sigma}}(t)= & \Gamma^{y}(C \Omega(t)+I)^{\top} D\left[\epsilon_{s}^{y}(t)\right] \\
& -\chi^{*} \Gamma^{y} \frac{\hat{\sigma}(t) \hat{\sigma}^{\top}(t)}{\hat{\sigma}^{\top}(t) \Gamma^{y} \hat{\sigma}(t)} \Gamma^{y}(C \Omega(t)+I)^{\top} D\left[\epsilon_{s}^{y}(t)\right]
\end{aligned}
$$

where $\chi^{*}$ denotes the indicator function

$$
\chi^{*}=\left\{\begin{array}{cc}
1 & \text { if }|\hat{\sigma}(t)|=M_{\sigma} \text { and } \\
& \hat{\sigma}^{\top}(t) \Gamma^{y}(C \Omega(t)+I)^{\top} D\left[\epsilon_{s}^{y}(t)\right]>0 . \\
0 & \text { otherwise. }
\end{array}\right.
$$

The dead-zone operator $D[\cdot]$ is given by

$$
D\left[\epsilon_{s}^{y}(t)\right]= \begin{cases}0 & \text { for } t<T_{d} \\ \epsilon_{s}^{y}(t) & \text { for } t \geq T_{d}\end{cases}
$$

where $T_{d}$ is the detection time of a fault $\left(T_{d}>T_{0}^{y}\right)$, which is defined as the first time instant such that $\left|\epsilon_{s}^{y}(t)\right|>\bar{\epsilon}_{s}^{y}(t)$, where $\bar{\epsilon}_{s}^{y}(t)$ is the detection threshold (to be specified later on). More specifically, $T_{d} \triangleq \inf \left\{t>0:\left|\epsilon_{s}^{y}(t)\right|>\bar{\epsilon}_{s}^{y}(t)\right\}$. Due to the presence of the modeling uncertainty and noise, the output estimation error deviates from zero even in the absence of faults. Therefore, the dead-zone operator is used in order to prevent adaptation whilst the residual is below the detection threshold. After a fault is detected, the deadzone operator is not necessary and can be disabled. By design, the adaptation for estimating the sensor fault in the estimation model (3)-(6) is initiated upon the detection of a fault. This is because, initially, $\hat{\sigma}(0)=0$ and from the deadzone operator definition, $D\left[\epsilon_{s}^{y}(t)\right]=0$ for $t<T_{d}$ (where the residual is bounded by the detection threshold), and hence $\hat{\sigma}(t)=\dot{\hat{\sigma}}(t)=0$ for $t<T_{d}$.

Prior to the occurrence of a fault, where the adaptation is not being used, the estimation model (3)-(6) is equivalent to

$$
\begin{aligned}
& \dot{\hat{x}}_{s}(t)=A \hat{x}_{s}(t)+f\left(\hat{x}_{s}(t), u(t)\right)+L \epsilon_{s}^{y}(t) \\
& \hat{y}_{s}(t)=C \hat{x}_{s}(t)
\end{aligned}
$$

and hence a suitable detection threshold $\bar{\epsilon}_{s}^{y}(t)$ which guarantees that $\left|\epsilon_{s}^{y}(t)\right| \leq \bar{\epsilon}_{s}^{y}(t)$ under healthy mode of operation (for all $t \leq T_{0}^{y}$ ) is given similarly as in [8]:

$$
\begin{aligned}
& \bar{\epsilon}_{s}^{y}(t) \triangleq|C|(E(t)+Z(t))+\bar{\xi}_{d}(t) \\
& \begin{aligned}
E(t) \triangleq \mu e^{-\rho t} \bar{x}_{d}+\int_{0}^{t} \mu e^{-\rho(t-\tau)}(\bar{\eta}(y(\tau), u(\tau)) \\
\left.+|L| \bar{\xi}_{d}(\tau)\right) \mathrm{d} \tau
\end{aligned} \\
& Z(t) \triangleq \mu \lambda_{f} \int_{0}^{t} E(\tau) e^{-\left(\rho-\mu \lambda_{f}\right)(t-\tau)} \mathrm{d} \tau,
\end{aligned}
$$

where $\mu, \rho$ are positive constants such that $\left|e^{A_{0} t}\right| \leq \mu e^{-\rho t}$ for all $t>0$ (note that, since $A_{0}$ is Hurwitz this is always feasible [22]) and also satisfy the condition $\rho-\mu \lambda_{f}>0$ (in order to guarantee the boundedness of the state estimation error), $\bar{x}_{d}(t)$ is a bound on the state in the region of operation $\mathcal{D}_{x}$, i.e. $|x(t)| \leq \bar{x}_{d}$ and, $\bar{\xi}_{d}(t)$ is a bound on the measurement noise, i.e. $|\xi(t)| \leq \bar{\xi}_{d}(t)$.

The nonlinear adaptive fault diagnosis scheme is robust in terms of the measurement noise and the modeling uncertainties satisfying Assumption 3. As a result, the designed detection threshold guarantees no false-positive alarms.

\section{Fault Detectability analysis}

In this section, a fault detectability condition is presented which constitutes a theoretical result that provides a quantitative characterization (and in non-closed form) of a class of faults detectable by the proposed scheme. The detectability condition given in the sequel is different from the one given in [8], due to different treatment of the mathematical analysis, and as a result the classes of detectable faults are different. It must be stressed though, that it cannot be determined if one condition is less conservative than the other, or if each condition contains a class of faults that are not contained in the other condition.

Theorem 1: Consider the nonlinear system (1), (2) with the nonlinear estimation model described by (3)-(6) and the detection scheme described by the output estimation error $\epsilon_{s}^{y}(t)$ and its detection threshold $\bar{\epsilon}_{s}^{y}(t)$ given by (10). A sensor fault occurring at time $T_{0}^{y}$ is detectable at some time $T_{d}>$ $T_{0}^{y}$ if the following inequality is satisfied

$$
\left|\sigma\left(T_{d}\right)\right|-|C|\left(K_{1}\left(T_{d}\right)+K_{2}\left(T_{d}\right)\right)>2 \bar{\epsilon}_{s}^{y}\left(T_{d}\right),
$$

$$
\begin{aligned}
& K_{1}(t) \triangleq \int_{0}^{t} \mu e^{-\rho(t-\tau)}|L \sigma(\tau)| \mathrm{d} \tau \\
& K_{2}(t) \triangleq \mu^{2} \lambda_{f} \int_{0}^{t} \int_{0}^{\tau} e^{-\rho(\tau-w)} e^{-\left(\rho-\mu \lambda_{f}\right)(t-\tau)}|L \sigma(w)| \mathrm{d} w \mathrm{~d} \tau .
\end{aligned}
$$

Proof: After a sensor fault occurs at $T_{0}^{y}$ and prior to the start of the adaptation (since it has not been detected yet), i.e. for $T_{0}^{y}<t<T_{d}$, the state estimation error $\epsilon_{s}^{x}(t)$ satisfies (the analysis is similar to the derivation of the detection threshold)

$\dot{\epsilon}_{s}^{x}(t)=A_{0} \epsilon_{s}^{x}(t)+\Delta f_{s}(t)+\eta(x(t), u(t), t)-L \xi(t)-L \sigma(t)$, 
where $\Delta f_{s}(t) \triangleq f(x(t), u(t))-f\left(\hat{x}_{s}(t), u(t)\right)$ and hence its solution is given by

$$
\begin{aligned}
\epsilon_{s}^{x}(t)= & e^{A_{0} t} \epsilon_{s}^{x}(0)+\int_{0}^{t} e^{A_{0}(t-\tau)}\left[\Delta f_{s}(\tau)\right. \\
& +\eta(x(\tau), u(\tau), \tau)-L \xi(\tau)-L \sigma(\tau)] d \tau .
\end{aligned}
$$

Following a similar procedure as in the derivation of the detection threshold, we obtain that

$$
\begin{aligned}
& \left|\epsilon_{s}^{x}(t)\right| \leq E^{\prime}(t)+Z^{\prime}(t), \\
& E^{\prime}(t) \triangleq \mu e^{-\rho t} \bar{x}_{d}+\int_{0}^{t} \mu e^{-\rho(t-\tau)}(\bar{\eta}(y(\tau), u(\tau)) \\
& \left.+|L| \bar{\xi}_{d}(\tau)+|L \sigma(\tau)|\right) \mathrm{d} \tau \\
& Z^{\prime}(t) \triangleq \mu \lambda_{f} \int_{0}^{t} E^{\prime}(\tau) e^{-\left(\rho-\mu \lambda_{f}\right)(t-\tau)} \mathrm{d} \tau .
\end{aligned}
$$

After some mathematical analysis (15), (16) are rewritten as

$$
\begin{aligned}
& E^{\prime}(t) \triangleq E(t)+K_{1}(t) \\
& Z^{\prime}(t) \triangleq Z(t)+K_{2}(t) .
\end{aligned}
$$

The output estimation error $\epsilon_{s}^{y}(t)$ satisfies

$$
\epsilon_{s}^{y}(t)=C \epsilon_{s}^{x}(t)+\xi(t)+\sigma(t)
$$

and by using (14), (17) and (18), (19) becomes

$$
\begin{aligned}
\left|\epsilon_{s}^{y}(t)\right| & \geq-|C|\left|\epsilon_{s}^{x}(t)\right|-|\xi(t)|+|\sigma(t)| \\
& \geq-|C|\left(E^{\prime}(t)+Z^{\prime}(t)\right)-\bar{\xi}_{d}(t)+|\sigma(t)| \\
& \geq-\bar{\epsilon}_{s}^{y}(t)-|C|\left(K_{1}(t)+K_{2}(t)\right)+|\sigma(t)| .
\end{aligned}
$$

For fault detection, the condition $\left|\epsilon_{s}^{y}(t)\right|>\bar{\epsilon}_{s}^{y}(t)$ at time $t=T_{d}$ must be satisfied and hence the fault detectability condition given in (13) is obtained.

Note that the detectability condition given in Theorem 1 is sufficient, but not a necessary one, and hence, the class of the detectable faults is possibly significantly larger. Intuitively, the magnitude of the fault needs to be sufficiently large so it can be distinguished from the effects of the modeling uncertainty and measurement noise.

\section{STABILITY AND APPROXIMATION PERFORMANCE}

In this Section, the stability properties and learning properties of the proposed adaptive estimation scheme are presented. Due to space constraints, the proof is omitted but, in general, it relies on similar results (i.e. [7], [14]), with necessary modifications carried out in order to address the difficulties incurred by the more general system formulation considered in this paper. The following theorem describes the properties of the proposed adaptive estimation scheme:

Theorem 2: In the event of a sensor fault that occurs at time $T_{0}^{y}$ and is detected at time $T_{d}>T_{0}^{y}$, the adaptive nonlinear estimation scheme described by (3), (4), (5), (6) under Assumptions $1-5$ and, by selecting the positive constants $\mu, \rho$ such that $\left|e^{A_{0} t}\right| \leq \mu e^{-\rho t}$ for all $t>0$ and $\rho-\mu \lambda_{f}>0$, guarantees that:

(a) The state estimation error $\epsilon_{s}^{x}(t)$, the output estimation error $\epsilon_{s}^{y}(t)$ and the sensor fault estimation vector $\hat{\sigma}(t)$ are uniformly bounded, i.e. $\epsilon_{s}^{x}(t), \epsilon_{s}^{y}(t), \hat{\sigma}(t) \in L_{\infty}$. (b) There exist a positive constant $q$ and a bounded function $v(t)$ such that, for all finite $t>T_{d}$ the output estimation error $\epsilon_{s}^{y}(t)$ satisfies:

$$
\int_{T_{d}}^{t}\left|\epsilon_{s}^{y}(\tau)\right|^{2} d \tau \leq q+4 \int_{T_{d}}^{t}|v(\tau)|^{2} d \tau .
$$

(c) In the absence of measurement noise (i.e. $\xi(t)=0$ ), if the bounded function $v(t)$ is square integrable (i.e. $\left.v(t) \in L_{2}\right)$, then $\lim _{t \rightarrow \infty} \epsilon_{s}^{y}(t)=0$.

Theorem 2 guarantees the uniform boundedness of the nonlinear adaptive estimation model, along with the state and output state estimation errors.

\section{SENSOR FAUlT ACCOMMODATION}

In this section, we propose a simple approach for accommodating sensor failures utilizing the sensor fault estimation vector $\hat{\sigma}$ which contains the estimation of the magnitude of the sensor faults $\sigma$. Note that, when a fault is detected the sensor fault estimation is initiated in order to learn the potential sensor faults, and thus, tries to estimate the sensor faults magnitudes for all sensors; healthy and faulty. So, the sensor fault estimation $\hat{\sigma}$ can be used to identify which of the sensors are healthy and which are faulty. Obviously for healthy sensors their respective sensor bias estimate should converge near zero, whereas for the faulty sensors their estimated bias will deviate significantly from zero. Based on this fact, the faulty sensors can be identified. More importantly though, and differently from other works, the estimation of the magnitudes of the sensor faults provides additional information that can be further exploited in fault accommodation schemes, through control reconfiguration.

When a fault is detected, it is desirable to compensate the fault effects to safeguard the operation of the system. In the case of sensor faults, these can affect the system performance through the feedback control law which is usually a function of the measurements and/or estimated states obtained from an observer scheme. Sensor faults also deteriorate state estimation schemes, and result in providing erroneous state estimates, which can also magnify the fault effect when these estimates are used in the feedback control law. Therefore, in order to increase the system performance, the correct output measurement must be reconstructed so that it can be used by the nonlinear observer and/or the feedback control law. To this end, the estimated sensor fault vector $\hat{\sigma}$ provides the necessary information for this reconstruction. Specifically, for a control law and/or any potential nonlinear observer used for state estimation which use the (potentially faulty) measurements $y$, a simple fault accommodation strategy is to replace the measurements $y$ with the compensated measurements $y-\hat{\sigma}$. This way, the control law and the nonlinear observer use the correct output information without the effect of the sensor bias fault $\sigma$ since it is canceled out by $\hat{\sigma}$ and therefore this fault compensation strategy allows for fault tolerant operation. Note that, due to the fact that $\hat{\sigma}(t)=0$ for $t \leq T_{d}$, there is no need for control reconfiguration or observer modification, and hence, both the 
control law and the nonlinear observer can be implemented to use the compensated values $y-\hat{\sigma}$ instead of $y$.

The effectiveness of this sensor fault accommodation strategy is demonstrated in the simulation results where a nonlinear observer is employed to provide state estimates and a feedback control law based on the measurements and the estimates is used for achieving the desired control task.

\section{Simulation RESUlts}

In this section, we consider a one-link manipulator with revolute joints actuated by a DC motor, as described in [23], [24]. The system model can be described by (1), (2) where $x=\left[x_{1}, x_{2}, x_{3}, x_{4}\right]$ is the system state comprising of: $x_{1}$ the angular rotation of the motor, $x_{2}$ the angular velocity of the motor, $x_{3}$ the angular position of the link and $x_{4}$ the angular velocity of the link and

$$
\begin{gathered}
A=\left[\begin{array}{cccc}
0 & 1 & 0 & 0 \\
-48.6 & -1.25 & 48.6 & 0 \\
0 & 0 & 0 & 1 \\
19.5 & 0 & -19.5 & 0
\end{array}\right], \eta=\left[\begin{array}{c}
0 \\
0 \\
0 \\
0.1 \sin \left(x_{3}\right)
\end{array}\right] \\
f=\left[\begin{array}{c}
0 \\
21.6 u \\
0 \\
-3.33 \sin \left(x_{3}\right)
\end{array}\right], C=\left[\begin{array}{llll}
1 & 0 & 0 & 0 \\
0 & 1 & 0 & 0
\end{array}\right] .
\end{gathered}
$$

The term $\eta(x)$ results by considering a $-3 \%$ inaccuracy in the mass. The initial conditions of the plant are set as $x=$ $\left[\begin{array}{llll}0 & 0 & 1 & 0\end{array}\right]$. The measurement noise is generated from a uniform distribution with $3 \%$ uncertainty of the state and the bound used for the task of fault detection is $\bar{\xi}_{d}=0.07$.

As stated in [23], one can measure only the motor position and velocity $\left(x_{1}\right.$ and $\left.x_{2}\right)$, but the measurement of the other states is non-trivial. That is why in our simulation we used this physical restriction, which adheres with the problem formulation (so that the nonlinearity function $f(x, u)$ comprises of unmeasurable variables), in contrary to previous works which required (some of) the other measurements $x_{3}$ and $x_{4}$ (i.e. [8], [14], [18]). Although the problem becomes more challenging now, the results also demonstrate the effectiveness of the proposed approach. The value of the Lipschitz constant for the nominal system is $\lambda_{f}=3.33$. In order to ease fault detection, we use a linear coordinate transformation $z=T x$, where $T=[1,1,1, \epsilon]$ with $\epsilon=$ 0.02 which effectively reduces the Lipschitz constant to $\epsilon \lambda_{f}$ [23] and therefore the bound on the modeling uncertainty is set as $\bar{\eta}=0.1 \epsilon$. The control task is to stabilize the system around the origin $x=0$. Spong [24] presents a nonlinear linearizing control which guarantees closed-loop stability, but the control law requires measurements of all the states. To address the unavailability of $x_{3}$ and $x_{4}$, we use the nonlinear observer designed in [23] to obtain their estimates and use these in the control law implementation. This observer is of the same type (Luenberger) that is being used in the current paper prior to the initiation of the learning, and therefore the designed nonlinear observer gain $L$ is perfectly suited also for the fault detection

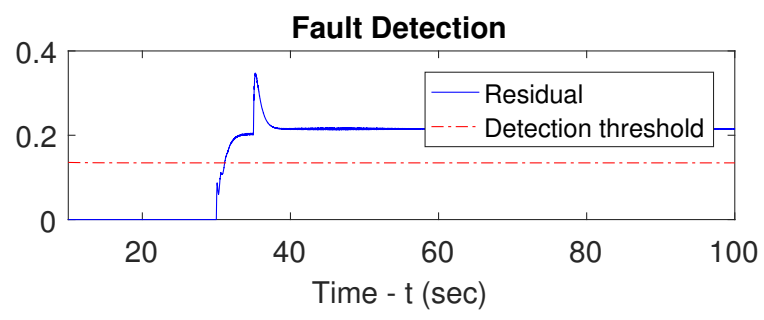

Fig. 1: Fault detection.
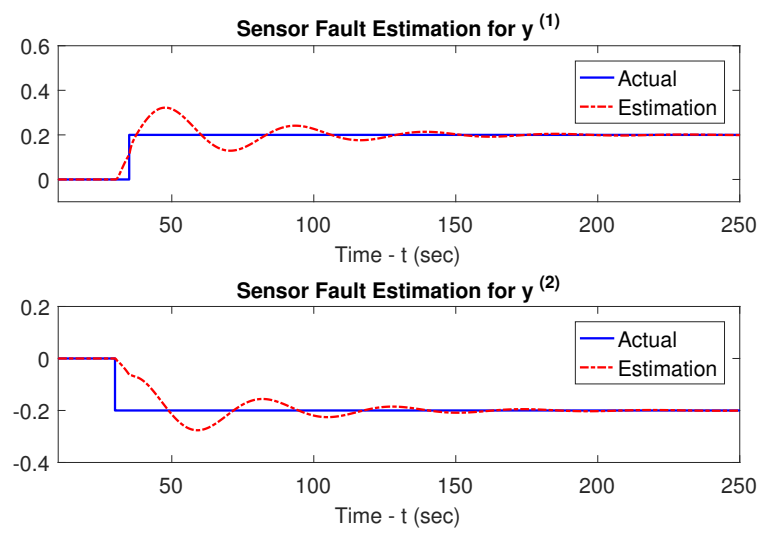

Fig. 2: Sensor fault estimation.

task. The observer gain matrix $L$ is given by [23]: $L=$ $\left[\begin{array}{lllll}0.830 & 0.451 ; 0.451 & 6.23 ; 0.823 & 1.307 ; 0.070 & 0.257\end{array}\right]$.

Through a suitable nonlinear state transformation and feedback linearization, the system is transformed in a 4-th order linear system in Brunovsky canonical form $\dot{w}_{i}=w_{i+1}$ $i=1,2,3$ and $\dot{w}_{4}=v$ and the linear state feedback law $v=-K w$ is then designed to place the poles of the closed loop system at $-10,-12,-14,-16$.

We consider the occurrence of two constant bias sensor faults which occur with $5 \mathrm{~s}$ difference as: $\sigma_{1}=0.2$ at $t=$ $35 \mathrm{~s}$ and $\sigma_{2}=-0.2$ at $t=30 \mathrm{~s}$ and affect the measurements $y_{1}$ and $y_{2}$ respectively (in addition to noise). Figure 1 shows the residual and detection thresholds signals and as can be seen the fault that appears first (in second sensor) is detected very fast at time $t=30.13 \mathrm{~s}$.

After the fault detection, the estimation of the sensor faults is initiated which adaptively tries to estimate magnitude of the potential sensor faults that have occurred. For this task we use the constant learning matrix $\Gamma^{y}=0.15 \mathbb{I}$ and the radius of the hypersphere for the sensor fault estimation is set to $M_{\sigma}=0.4$. The results are shown in Figure 2, in which it can be seen that both sensor faults are estimated correctly.

Moreover, in order to demonstrate the effectiveness of the proposed approach for the fault accommodation task, we demonstrate two cases. In the first case, adaptation is not used, and therefore the nonlinear observer and feedback control law rely on the (faulty) measurements $y_{1}$ and $y_{2}$. In the second case, adaptation is used and the proposed fault accommodation scheme is implemented so that the estimates of the potential sensor faults are used in order to accommodate the sensor faults. Hence, in the second 
System states with and without without fault accommodation
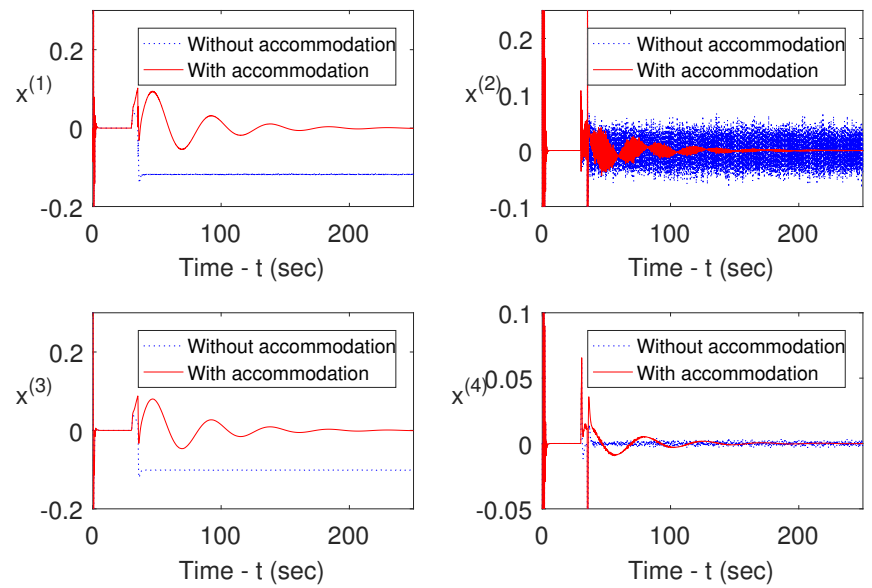

Fig. 3: System states with and without fault accommodation. When fault accommodation is used, the control task of stabilizing the system states at the origin is maintained.

case the nonlinear observer and feedback control use the accommodated values $y_{1}-\hat{\sigma}_{1}$ and $y_{2}-\hat{\sigma}_{2}$. Note that in all cases, the feedback control law makes use of the estimated states $x_{3}$ and $x_{4}$ obtained from the nonlinear observer. Figure 3 shows the actual system states in the two cases. Note that, prior to the fault occurrence, in both cases the control objective of maintaining the states at the origin is achieved. After the fault occurrence though, in the first case (no fault accommodation) all the system states except $x_{4}$ deviate significantly from zero, whereas in the second case (with fault accommodation) all system states converge to zero and therefore the control objective of maintaining the states at the origin is achieved.

\section{CONCLUSION}

In this paper, an adaptive sensor fault diagnosis and accommodation scheme for multiple sensor bias faults is designed for a class of input-output nonlinear uncertain systems, that is robust in terms of modeling uncertainty and measurement noise. The nonlinear estimation scheme utilizes an adaptive scheme that is initiated upon the detection of a fault for estimating the sensor bias faults. The robustness and stability properties of the proposed scheme are presented and, a fault detectability condition is derived. The estimation of the magnitude of the sensor bias faults allows the identification of the faulty sensors and can also be used for fault accommodation purposes. Future research efforts will be devoted in designing a comprehensive framework for fault diagnosis for both process and sensor faults.

\section{REFERENCES}

[1] V. Venkatasubramanian, R. Rengaswamy, K. Yin, and S. Kavuri, "A review of process fault detection and diagnosis Part I: Quantitative model-based methods," Computers \& Chemical Engineering, vol. 27, no. 3, pp. 293-311, Mar. 2003.

[2] I. Hwang, S. Kim, Y. Kim, and C. E. Seah, "A Survey of Fault Detection, Isolation, and Reconfiguration Methods," IEEE Transactions on Control Systems Technology, vol. 18, no. 3, pp. 636-653, May 2010.
[3] M. Blanke, M. Kinnaert, J. Lunze, and M. Staroswiecki, Diagnosis and Fault-Tolerant Control, 2nd ed. Springer Verlag, 2010.

[4] X. Zhang, M. M. Polycarpou, and T. Parisini, "A robust detection and isolation scheme for abrupt and incipient faults in nonlinear systems," IEEE Transactions on Automatic Control, vol. 47, no. 4, pp. 576-593, 2002.

[5] C. Keliris, M. M. Polycarpou, and T. Parisini, "A Distributed Fault Detection Filtering Approach for a Class of Interconnected ContinuousTime Nonlinear Systems," IEEE Transactions on Automatic Control, vol. 58, no. 8, pp. 2032-2047, 2013.

[6] M. M. Polycarpou and A. Helmicki, "Automated fault detection and accommodation: A learning systems approach," IEEE Transactions on Systems, Man and Cybernetics, vol. 25, no. 11, pp. 1447 - 1458, 1995.

[7] C. Keliris, M. M. Polycarpou, and T. Parisini, "An Integrated Learning and Filtering Approach for Fault Diagnosis of a Class of Nonlinear Dynamical Systems," IEEE Transactions on Neural Networks and Learning Systems, vol. 28, no. 4, pp. 988-1004, 2017.

[8] V. Reppa, M. Polycarpou, and C. Panayiotou, "Adaptive Approximation for Multiple Sensor Fault Detection and Isolation of Nonlinear Uncertain Systems," IEEE Transactions on Neural Networks and Learning Systems, vol. 25, no. 1, pp. 137-153, 2014.

[9] M. Demetriou, "Robust adaptive techniques for sensor fault detection and diagnosis," in Proceedings of Control and Decision Conference, 1998, pp. $1143-1148$.

[10] A. B. Trunov and M. M. Polycarpou, "Automated fault diagnosis in nonlinear multivariable systems using a learning methodology." IEEE Transactions on Neural Networks, vol. 11, no. 1, pp. 91-101, Jan. 2000

[11] Bin Jiang and F. Chowdhury, "Fault estimation and accommodation for linear MIMO discrete-time systems," IEEE Transactions on Control Systems Technology, vol. 13, no. 3, pp. 493-499, may 2005.

[12] D. Du, B. Jiang, and P. Shi, "Sensor fault estimation and accommodation for discrete-time switched linear systems," IET Control Theory \& Applications, vol. 8, no. 11, pp. 960-967, 2014.

[13] R. Rajamani and A. Ganguli, "Sensor fault diagnostics for a class of non-linear systems using linear matrix inequalities," International Journal of Control, vol. 77, no. 10, pp. 920-930, 2004.

[14] X. Zhang, T. Parisini, and M. M. Polycarpou, "Sensor bias fault isolation in a class of nonlinear systems," IEEE Transactions on Automatic Control, vol. 50, no. 3, pp. 370-376, Mar. 2005.

[15] X.-G. Yan and C. Edwards, "Sensor fault detection and isolation for nonlinear systems based on a sliding mode observer," International Journal of Adaptive Control and Signal Processing, vol. 21, no. 8-9, pp. 657-673, oct 2007.

[16] V. Reppa, M. M. Polycarpou, and C. G. Panayiotou, "Decentralized Isolation of Multiple Sensor Faults in Large-Scale Interconnected Nonlinear Systems," IEEE Transactions on Automatic Control, vol. 60 , no. 6, pp. 1582-1596, 2015

[17] M. J. Daigle, X. D. Koutsoukos, and G. Biswas, "Distributed diagnosis in formations of mobile robots," IEEE Transactions on Robotics, vol. 23, no. 2, pp. 353-369, 2007.

[18] X. Zhang, "Sensor Bias Fault Detection and Isolation in a Class of Nonlinear Uncertain Systems Using Adaptive Estimation," IEEE Transactions on Automatic Control, vol. 56, no. 5, pp. 1220-1226, 2011

[19] Q. Zhang and X. Zhang, "Distributed sensor fault diagnosis in a class of interconnected nonlinear uncertain systems," Annual Reviews in Control, vol. 37, no. 1, pp. 170-179, 2013.

[20] P. M. Papadopoulos, V. Reppa, M. M. Polycarpou, and C. G. Panayiotou, "Distributed adaptive estimation scheme for isolation of sensor faults in multi-zone HVAC systems," in IFAC-PapersOnLine, vol. 28, no. 21. Elsevier Ltd., 2015, pp. 1146-1151.

[21] A. Vemuri and M. Polycarpou, "Robust nonlinear fault diagnosis in input-output systems," International Journal of Control, vol. 68, no. 2, pp. 343-360, 1997.

[22] P. Ioannou and J. Sun, Robust adaptive control. Prentice Hall, 1996.

[23] R. Rajamani and Y. Cho, "Existence and design of observers for nonlinear systems: relation to distance to unobservability," International Journal of Control, vol. 69, no. 5, pp. 717-731, 1998.

[24] M. W. Spong, "Modeling and control of elastic joint robots," Journal of Dynamic Systems, Measurement, and Control, vol. 109, no. 4, pp. 310-319, 1987. 